\title{
Il medico internista e l'Ortopedia: quali competenze, quali modelli?
}

\section{Internists and Orthopedics: which competences and models are needed?}

L'aumento dell'età media dei pazienti ospedalizzati, l'aumento delle comorbilità e la conseguente maggior complessità di cura costituiscono fattori di rischio per i pazienti ricoverati in ospedale. L'obiettivo qualificante nel concepire il ruolo nuovo del moderno ospedale è quello di mettere al centro della struttura il paziente, con i suoi bisogni assistenziali, attraverso percorsi certi e sicuri, garantiti da un'organizzazione volta a favorire un'assistenza continua e personalizzata, nel rispetto dell'appropriatezza nell'uso delle risorse. In questo senso, perno dell'organizzazione diventa la Medicina Interna, capace di analizzare lo stato di salute nel suo complesso garantendo una risposta globale ai bisogni e rispettando la centralità del paziente. Il paziente anziano è, infatti, per lo più polipatologico, spesso con difficoltà di movimento e dipendenza funzionale, talora malnutrito e fragile, intendendo per fragilità una sorta di vulnerabilità fisiologica correlata all'età. Appare naturale, così, affidare allo specialista internista il malato nella sua interezza, riservando il ruolo dello specialista alla patologia d'organo evidenziata, con un interscambio nell'utilizzo dei letti in rapporto alle esigenze del caso.

L'opportunità di introdurre una figura professionale specialista in Medicina Interna per migliorare l'efficienza delle cure prestate, nonché la qualità, è alla base del movimento degli "hospitalists", sviluppatosi circa 10 anni fa negli Stati Uniti; gli "hospitalists" sono definiti come "medici il cui principale interesse è il trattamento internistico generale [general medical care] del paziente ospedalizzato" [1]. Tale figura professionale, pur basata su modelli diversi, è rappresentata da medici di Medicina Interna che prestano almeno il $25 \%$ della propria attività lavorativa nell'assistenza internistica di pazienti ricoverati [2].

Vari studi americani hanno osservato come l'attività propria del medico internista ospedaliero (hospitalist) comporti grandi benefici sia per i pazienti sia per le strutture di ricovero: da un lato, sono garantite l'unicità e la globalità del trattamento, senza eccessi di dispersione in particolarismi settoriali; dall'altro, si ha un notevole risparmio per le Aziende Ospedaliere, che ottengono il trattamento migliore dei pazienti con il più favorevole rapporto costo/beneficio in termini tanto di salute quanto di economicità.

Sulla scorta dell'esperienza statunitense, e considerata la crescente complessità assistenziale dei pazienti ricoverati nei reparti di Ortopedia, in larga parte legata all'aumento dell'età e delle comorbilità, appare logico ipotizzare che il processo di cura tradizionale, con lo specialista che eventualmente si avvale di una consulenza internistica, possa essere sostituito da un modello in cui uno specialista in Medicina Interna sia integrato all'interno della Divisione Chirurgica e collabori al processo assistenziale facendosi carico direttamente delle problematiche di ordine medico del paziente. Risulterebbe più facile, inoltre, garantire una continuità assistenziale in merito a eventuali patologie preesistenti, sia a momento del ricovero sia alla dimissione.

Gli studi disponibili in letteratura in merito agli effetti delle presenza di un hospitalist sono stati oggetto di una recente revisione sistematica [3].

In questi lavori è stato evidenziato, in generale, un miglioramento della degenza ospedaliera, con una riduzione dei costi; gli indici di migliore qualità della cura, invece, sono stati riportati in modo meno costante. In particolare, sono emersi un minor tasso di re-ricoveri per i pazienti affetti da scompenso cardiaco e dolore toracico a basso rischio, e una migliore appropriatezza nella prescrizione di farmaci nello scompenso cardiaco e nella profilassi tromboembolica nei pazienti con polmonite acquisita in comunità.

Per quanto attiene alle esperienze in campo ortopedico, Huddleston et al. [4] hanno arruolato in uno studio randomizzato 526 pazienti a elevato rischio di complicanze postoperatorie sottoposti a intervento chirurgico di artroplastica di anca o ginocchio e seguiti da un hospitalist oppure dal chirurgo ortopedico con eventuali consulenti; gli outcome misurati erano la durata della degenza, il tasso di complicanze intraoperatorie, la soddisfazione del paziente e del personale medico e i costi. I pazienti seguiti dall'hospitalist avevano un minor tasso di complicanze postoperatorie, mentre i costi assistenziali non variavano; anche la durata della degenza osservata era invariata. Gli autori concludono 
che il modello dell'hospitalist riduce le complicanze minori, non riduce i costi né la durata della degenza postoperatoria, ma risulta particolarmente soddisfacente per gli operatori (medici e infermieri).

Roy et al. [5] hanno studiato retrospettivamente 118 pazienti con frattura di femore seguiti da un hospitalist o dal chirurgo ortopedico con eventuale richiesta di consulto preoperatorio; il loro studio conferma una riduzione della degenza e dei costi, peraltro non significativa, e un accesso più precoce alla camera operatoria.

Phy et al [6] hanno arruolato nell'arco di 2 anni 466 pazienti di età superiore a 65 anni e affetti da frattura di femore; gli outcome sono stati misurati in due periodi consecutivi di 12 mesi ciascuno, prima e dopo l'introduzione dell'hospitalist. Gli autori evidenziano che il modello dell'hospitalist riduce sia il tempo tra l'accettazione e la correzione chirurgica sia la degenza postoperatoria; gli esiti a distanza di un anno nella stessa popolazione dimostrano che non vi è differenza nella sopravvivenza a un anno [7].

Presso l'Azienda Ospedaliera "Ospedale Niguarda Ca' Granda" di Milano, il modello dell'hospitalist è stato applicato presso la Divisione di Ortopedia e Traumatologia, nella quale vengono eseguiti circa 1.200 interventi chirurgici l'anno; la Divisione comprende 50 posti letto e vi lavorano 18 chirurghi.

Dall'ottobre 2007 un internista esperto (specialista in Medicina Interna con anzianità di servizio di circa 20 anni, di cui 10 in Medicina d'Urgenza) ha iniziato a prestare la sua attività presso la Divisione. Per i primi 2 mesi ha studiato il modello organizzativo senza apportare modifiche. Dal gennaio 2008 il suo compito è consistito nel valutare tutti i pazienti ricoverati nel reparto (entro 48 ore dal ricovero), gestirne le problematiche mediche, richiedere le consulenze specialistiche e collaborare a tutti gli effetti alla gestione del paziente.

La sua presenza è stata garantita per 38 ore la settimana, distribuite su 5-6 giorni la settimana.

Il modello di co-management ha determinato una riduzione dei giorni di degenza $(17,5$ giorni/paziente vs 15,4$)$ soprattutto postoperatoria $(10,5$ vs 8,8$)$, con un minor numero di trasferimenti in altri reparti internistici o ad alta intensità di cura $(35$ vs $20, p>0,1)$. Si è avuta una netta diminuzione degli esami radiologici richiesti, degli esami biochimici e delle consulenze specialistiche $(0,4$ consulenze/paziente vs 0,3 ). Si è evidenziata una riduzione sia di alcune complicanze associate alla qualità della cura prestata (scompenso metabolico, insufficienza respiratoria, trombosi venosa profonda ed embolia polmonare, sepsi postoperatoria) sia dei trasferimenti verso la Terapia Intensiva e la Medicina Interna. Inoltre, sono migliorati alcuni indicatori di processo quali l'adeguatezza della profilassi antibiotica e della profilassi antitrombotica.

Anche l'articolo di Carlo Frondini e Maria Lia Lunardelli ("Ortogeriatria: un nuovo modello di assistenza ai pazienti anziani con frattura di femore e comorbilità") presenta un progetto volto a delineare un approccio integrato multidisciplinare e multiprofessionale in cui gli attori sono il geriatra, l'ortopedico, il fisiatra, ponendo il paziente al centro dell'assistenza, con risultati incoraggianti in termini di riduzione della degenza media e della mortalità ospedaliera.

Una rassegna sistematica [8] ha di recente fatto il punto sui trial clinici attualmente disponibili riguardanti i

Tabella 1 Modelli ortogeriatrici.

\begin{tabular}{|c|c|c|c|}
\hline & Tipologia & Pubblicazioni & Outcome \\
\hline \multirow{3}{*}{$\begin{array}{l}\text { Fase acuta } \\
\text { ospedaliera }\end{array}$} & $\begin{array}{l}\text { Assistenza multidisciplinare } \\
\text { nella fase acuta }\end{array}$ & $\begin{array}{l}3 \text { studi randomizzati } \\
\text { anche se con diversi } \\
\text { componenti del team }\end{array}$ & $\begin{array}{l}\text { Con questo modello sembra } \\
\text { dimostrata la riduzione delle } \\
\text { complicanze e della mortalità }\end{array}$ \\
\hline & $\begin{array}{l}\text { Riabilitazione accelerata } \\
\text { con comanagement } \\
\text { nell'immediato } \\
\text { postoperatorio }\end{array}$ & & $\begin{array}{l}\text { Effetti soprattutto sulla durata } \\
\text { della degenza e sul recupero } \\
\text { funzionale precoce }\end{array}$ \\
\hline & $\begin{array}{l}\text { Modello assistenziale a tipo } \\
\text { "hospitalist" (modello } \\
\text { di organizzazione ospedaliera } \\
\text { tipicamente americano } \\
\text { che nei pazienti chirurgici } \\
\text { è da considerarsi a tutti } \\
\text { gli effetti comanagement) }\end{array}$ & & $\begin{array}{l}\text { I dati sui pazienti ortopedici } \\
\text { hanno evidenziato soprattutto } \\
\text { il miglioramento degli aspetti } \\
\text { gestionali ma non benefici } \\
\text { sugli outcome maggiori }\end{array}$ \\
\hline Fase subacuta & $\begin{array}{l}\text { Comanagement ortogeriatrico } \\
\text { della fase subacuta riabilitativa } \\
\text { in strutture di lungodegenza } \\
\text { o di cure intermedie }\end{array}$ & $\begin{array}{l}\text { È uno dei modelli più } \\
\text { studiati con ben } 11 \text { trial } \\
\text { RCT e } 2 \text { metanalisi } \\
\text { pubblicate }\end{array}$ & $\begin{array}{l}\text { Per quanto sia stato evidenziato } \\
\text { un certo miglioramento } \\
\text { degli outcome funzionali } \\
\text { non riduce la mortalità }\end{array}$ \\
\hline \multirow[t]{2}{*}{ Fase riabilitativa } & $\begin{array}{l}\text { Comanagement ortogeriatrico } \\
\text { unico dalla fase acuta } \\
\text { al completamento della fase } \\
\text { riabilitativa }\end{array}$ & $\begin{array}{l}\text { Realizzato in alcune } \\
\text { realtà territoriali ma } \\
\text { non sperimentato } \\
\text { su larga scala }\end{array}$ & \\
\hline & $\begin{array}{l}\text { Modelli di riabilitazione } \\
\text { domiciliare }\end{array}$ & & $\begin{array}{l}\text { Attuabili su un numero limitato } \\
\text { di pazienti ma con forte impatto } \\
\text { sulla qualità della vita }\end{array}$ \\
\hline
\end{tabular}


modelli organizzativi ortogeriatrici (tabella 1): l'estrema eterogeneità dei modelli associata alla scarsità di studi controllati e randomizzati non consente di trarre ancora conclusioni definitive sulla validità del comanagement ortogeriatrico.

Ciononostante sono sempre più numerosi i dati che indicano un evidente beneficio in termini di durata della degenza, di riduzione delle complicanze e della mortalità.

Le review Cochrane non consentono di individuare un modello gestionale di riferimento, tuttavia l'elaborazione di efficaci indicatori di outcome permetterebbe di verificare la congruenza dell'assunto di base dei modelli proposti, vale a dire che questi modelli renderebbero disponibili elementi conoscitivi necessari per la realizzazione di interventi migliorativi nonché di economie di gestione.

In conclusione, le esperienze descritte in letteratura confermano l'approccio multiprofessionale e interdisciplinare e dimostrano che i modelli non solo sono introducibili all'interno della realtà italiana, ma si associano a un miglioramento della qualità delle cure erogate grazie a una maggiore efficienza.

\section{Bibliografia}

[1] Wachter RM. The evolution of the hospitalist model in the United States. Med Clin North Am 2002;86(4):687-706.
[2] Wachter RM. An introduction to the hospitalist model. Ann Intern Med 1999;130(4 Pt 2):338-42.

[3] Peterson MC. A systematic review of outcomes and quality measures in adult patients cared for by hospitalists vs nonhospitalists. Mayo Clin Proc 2009;84(3):248-54.

[4] Huddleston JM, Long KH, Naessens JM, et al., Hospitalist-Orthopedic Team Trial Investigators. Medical and surgical comanagement after elective hip and knee arthroplasty: a randomized, controlled trial. Ann Intern Med 2004;141(1):28-38.

[5] Roy A, Heckman MG, Roy V. Associations between the hospitalist model of care and quality-of-care-related outcomes in patients undergoing hip fracture surgery. Mayo Clin Proc 2006;81(1): $28-31$.

[6] Phy MP, Vanness DJ, Melton 3rd LJ, et al. Effects of a hospitalist model on elderly patients with hip fracture. Arch Intern Med 2005;165(7):796-801.

[7] Batsis JA, Phy MP, Joseph Melton 3rd L, et al. Effects of a hospitalist care model on mortality of elderly patients with hip fractures. J Hosp Med 2007;2(4):219-25.

[8] Chong CP, Savige J, Lim WK. Review article. Australas J Ageing 2009 Dec;28(4):171-6.

Fabrizio Colombo

Direttore Dipartimento Medico Polispecialistico, AO "Ospedale Niguarda Ca' Granda", Milano E-mail: fabrizio.colombo@ospedaleniguarda.it 